\title{
Effect of fiber loading directions on the low cycle fatigue of intraply carbon- Kevlar reinforced epoxy hybrid composites
}

\begin{abstract}
Effect of fiber orientation on tensile and low cycle fatigue of intraply hybrid composites was investigated. In this work, intraply carbon/Kevlar fabric/epoxy hybrid composites were tested for static and cyclic loadings at $0^{\circ}, 45^{\circ}$ and $90^{\circ}$ corresponding to carbon, off-axis and Kevlar fiber directions. Ambient fatigue tests were performed at stress levels established from static tests. Tensile results showed the best strength and modulus was in the carbon direction followed by Kevlar direction. At $45^{\circ}$, high ductility was observed associated to fiber rotation. Fatigue linear regression lines indicated a slower degradation rate in the Kevlar loading direction compared to the carbon loading direction. Large fatigue scatter in the Kevlar direction suggested two distinct phases of fatigue attributed to the stiffening of Kevlar fibers that further led to lower fatigue degradation. Investigation on residual strength of run-out samples and fracture modes were also presented with interesting findings.
\end{abstract}

